\title{
The pathogens and curative effects analysis of perianal abscess of infants under 3 months
}

\author{
Yimin Zhu, Fuying Xu
}

Department of General Surgery, The First Affiliated Hospital of Lishui University, Lishui, China. E-mail: xufuying@lsu.edu.cn Received: 18th December 2017, Revised: 2nd March 2018, Accepted: 27th March 2018

SUMMARY: Zhu Y, Xu F. The pathogens and curative effects analysis of perianal abscess of infants under 3 months. Turk J Pediatr 2019; 61: 40-43.

In order to guide clinical treatment for perianal abscess of young infants, the characteristics of pathogens and curative effects analysis were conducted. Bacterial culture results, antibiotics susceptibility tests and curative effects of abscess incision were retrospectively analyzed in 66 cases of perianal abscess of infants under 3 months. There were 48 cases of Klebsiella pneumoniae, 7 cases of Staphylococcus, 6 cases of Escherichia coli, 5 cases of Proteus in the pathogen culture results. Klebsiella pneumoniae, the predominant pathogen, was susceptible to most antibiotics, especially to imipenem, cefoperazonesulbactam and amikacin with low drug resistance rates. However, high drug resistance rates were found to ampicillin and nitrofurantion. After abscess incision, the complication rate of anal fistula was $6.6 \%$ in infants under 3 months and $60.3 \%$ in the adult group. There was significant difference $P<0.01$. In conclusion, Klebsiella pneumoniae was the most common pathogen in perianal abscess of infants under 3 months and was commonly resistant to ampicillin and nitrofurantion. Since perianal abscess of infants under 3 months is a self-limited disorder, simple surgical intervention and synchronous sensitive antibiotic administration are suggested as the optimal management.

Key words: infant, periana1 abscess, germicu1ture, curative effect.

The characterization of perianal abscess in infants is descriptive, based mainly on clinical features. They are usually superficial entities close to the anus; however the variability in size, precise location, and relation to the anal sphincter system, in addition to the deficiency of information on underlying fistulae and the lack of contemporaneous ultrasound or magnetic resonance imaging studies, have contributed to the confusion and variability regarding treatments. ${ }^{1,2}$ Thereby, until now, perianal abscess remains unclassified, without unanimous data regarding treatment and surgical techniques that should be adopted. Recurrence or development of fistula-in-ano rates after initial treatment of perianal abscess are reported in 0 to $85 \%$ of cases. ${ }^{3}$ This wide range may be due to therapeutic differences. We found that perianal abscess was common in infants less than 3 months of age and had an overwhelming male predominance. To the best of our knowledge, no systematic study has reported its etiology and the optimal management for this condition remained obscure. A few case reports and case series of young infant perianal abscess (YIPA) were found and various approaches have been suggested for the management of YIPA; however, there were no consensus guidelines or recommendations of high efficacy approach for this condition 4 . In order to identify the characteristics of pathogens and find an optimal therapeutic protocol for YIPA, the clinical data of the 66 YIPA cases as well as 58 cases of adult perianal abscess were retrospectively analyzed by comparing both in bacterial culture results, antibiotics susceptibility tests and curative effects of abscess incision. The clinical features and outcome of YIPA were summarized and reported as the following.

\section{Data and method}

Clinical data

Patients were included in the study after the necessary permission had been acquired 
Table I. Bacterial Culture Results of Perianal Abscess.

\begin{tabular}{|c|c|c|}
\hline \multirow[b]{2}{*}{ Pathogens } & \multicolumn{2}{|c|}{ Positive cases/ Rate } \\
\hline & YIPA group & Adult group \\
\hline Klebsiella pneumoniae & $48 \quad(72.7 \%)$ & $2(3.4 \%)$ \\
\hline Escherichia coli & $6 \quad(9.1 \%)$ & $51(87.9 \%)$ \\
\hline Proteus & $5 \quad(7.6 \%)$ & $2(3.4 \%)$ \\
\hline Staphylococcus & $7 \quad(10.6 \%)$ & $1 \quad(1.7 \%)$ \\
\hline Streptococcus & $0 \quad(0 \%)$ & $2(3.4 \%)$ \\
\hline
\end{tabular}

from the ethics committee of our hospital in Feb. 2015 (No. 20150203). Informed consent was acquired from the patients' families. A retrospective study was conducted for review of in-hospital medical charts and outpatient charts of perianal abscess from Sep. 2013 to Dec. 2014. The YIPA group consisted of 66 cases, included 63 males and 3 females. They were between 22-90 days old (average 38.6 days), 15 of them under 30 days, 42 of them 31-60 days and 9 of them 61-90 days. The high incidence of period was around 30 days. Perianal abscess sites of 26 cases were at three o'clock in lithotomy position, 30 cases at nine o'clock and 10 cases at six o'clock. Perianal skin red swelling, induration or fluctuation by touching were the clinical signs of physical inspection. The duration were 1-5 days (average 1.9 days). The control group (Adult group) included 58 cases of adults, 52 males and 6 females, 18-69 years old (average 36.8 years), duration 1-6 days (average 2.1 days). The abscess sites of 20 cases were at three o'clock in lithotomy position, 21 cases at nine o'clock and 17 cases at six o'clock. All the cases in both groups were diagnosed as simple perianal subcutaneous un-ruptured abscess. Those cases with concurrent disease such as congenital heart disease, immunodeficiency disease, diabetes, tuberculosis, Crohn disease or malnutrition were excluded from this study. Abscess incision and drainage were performed on each case. After regional anesthesia, at the obvious fluctuation site of the perianal abscess a radial incision was made, which had the length proximity to the diameter of the abscess. Pus and necrotic tissue were completely removed and a vaseline gauze was stuffed into the abscess. Then, the drainage gauze was changed daily. Pus was sent for bacterial culture and antibiotic susceptibility testing. At initial treatment, we empirically chose Ceftriaxone or Piperacillin-tazobactam intravenous drip as the first-opted in view of their broad spectrum covering Gram-negative and positive bacterium and few resistance incidence. If their susceptibility test indicated resistance, they would be replaced by the sensitive one. After infusion of sensitive antibiotic for 3-5 days, the condition could be controlled. Subsequently, the oral antibiotics such as cefixime or cefaclor was taken for 7-10 days. By following for 6 months, the outcome of abscess was tracked.

\section{Statistic method}

The data in each group were entered and analyzed using SPSS 10.0 software for Windows XP. Chi-square test was performed. A value of $P<0.05$ was considered statistically significant.

\section{Results}

There were no significant difference in the general data except age between the groups $(P>0.05)$. We found a significant predominance of non-enteric flora such as Klebsiella pneumonia, Staphylococcus in the YIPA group. However, enteric flora such as E.coli, Proteus were the most common pathogens in the adult group. Table I shows the pathogen culture results of both groups.

Pathogens of all the 124 cases in bacterial culture results of both YIPA and adult groups were consisted of Klebsiella pneumonia, Staphylococcus, E.coli, Proteus and Streptococcus. Their antibiotic resistance rates to common antibiotics are showed in the Table II. Klebsiella pneumonia was susceptible to most antibiotics, especially to Imipenem, Cefoperazone-sulbactam and Amikacin with low drug resistance rate. However, high 
Table II. Antibiotic Resistance Rates of Pathogens in Bacterial Culture of Perianal Abscess (\%).

\begin{tabular}{lccccc}
\hline Antibiotics & $\begin{array}{c}\text { Klebsiella } \\
\text { pneumoniae }\end{array}$ & Staphylococcus & $\begin{array}{c}\text { Escherichia } \\
\text { coli }\end{array}$ & Proteus & Streptococcus \\
\hline Cefoxitin & 12.0 & & 31.5 & 28.6 & \\
Ceftazidime & 12.0 & & 39.3 & 28.6 & \\
Imipenem & 0.0 & & 0.0 & 0.0 & \\
Aztreonam & 16.0 & & 8.8 & 14.3 & \\
Cefoperazone-sulbactam & 6.0 & & 3.5 & 0.0 & \\
Piperacillin-tazobactam & 10.0 & 12.5 & 56.5 & 28.6 & 0.0 \\
Levofloxacin & 6.0 & 50.0 & 40.3 & 14.3 & 0.0 \\
Cefazolin & 22.0 & 62.5 & 42.0 & 42.8 & 0.0 \\
Ceftriaxone & 14.0 & 25.0 & 29.5 & 14.3 & 0.0 \\
Nitrofurantoin & 92.0 & 50.0 & 59.5 & 57.1 & 50.0 \\
Amikacin & 4.0 & 12.5 & 8.8 & 0.0 & 0.0 \\
Gentamycin & 22.0 & 50.0 & 56.0 & 57.1 & 50.0 \\
Ampicillin & 96.0 & 75.0 & 70.0 & 71.4 & 50.0 \\
SMZ & 18.0 & 37.5 & 73.5 & 71.4 & 100.0 \\
Penicillin & & 100.0 & & & 50.0 \\
\hline
\end{tabular}

The antibiotic resistance rates of pathogens in bacterial culture of all the 124 cases in YIPA and adult groups to common antibiotics.

resistance rates were found to Ampicillin and Nitrofurantion.

Abscess incision and drainage were performed and each case was followed for six months. The complication of anal fistula occurred in 4 cases out of the 66 cases making up $6.6 \%$ in the YIPA group and 35 cases out of the 58 cases making up $60.3 \%$ in the adult group. There was significant difference $P<0.01$.

\section{Discussion}

Infant with perianal abscess seems to be a peculiar population. ${ }^{2}$ We observed that infant perianal abscess frequently occurred in male infants under 3 months of age in our clinical practice. The recurrence of an abscess or the development of a fistula, after the incision of perianal abscess, is rare in infants when compared with adults. There are a number of proposed theories to explain the etiology and perianal abscess formation in infants; however, the true pathophysiologic mechanism remains uncertain. The prevailing theories are the congenital theory, which implicates the excess production of androgens, and the one which is based on the existence of abnormal anal crypts. ${ }^{2-7}$
The option of conservative treatment or surgery is often determined depending on personal preference of physicians rather than consensus guidelines. Surgical intervention includes incision and drainage, active probing for underlying fistulae and fistulotomy. Due to the weak anal sphincter and tender tissue of young infants, the structure of the anal canal could be damaged by active probing or fistulotomy and caused a false canal. Thread-drawing and fistulotomy could frequently harm the function of anal sphincter resulting in anus leakage and incontinence. Therefore, the suitable approach to manage YIPA is abscess incision. Even if an anal fistula formed later, it could be excised during adulthood. ${ }^{8,9}$ In our study all the cases were followed. In the YIPA group, incisions of 62 cases healed within four weeks, and 4 cases experienced disunion for a duration of about six months. In the adult group, however, complications of anal fistula occurred in $60.3 \%$ of the cases after subcutaneous abscess incision, and was finally cured by fistulotomy. In general, the underlying fistulaes tend to be simple, low and direct in infants but they are not the same in adults. For the YIPA, incision and drainage are easy, safe and curative approach with low recurrence incidence. 
Our results indicated the diversity of microorganisms in relation to different populations of patients. We differentiated the microorganisms in pus culture as enteric flora, non-enteric flora and skin flora bacteria. The most common pathogen was Klebsiella pneumonia in the YIPA group, while Escherichia coli was predominant in the adult group. If enteric flora is present in the pus of perianal abscess, a fistula may form. ${ }^{10,11}$ Antibiotic susceptibility tests showed that Klebsiella pneumonia was susceptible to most antibiotics, but highly resistant to Ampicillin and Nitrofurantion. Ceftriaxone or Piperacillintazobactam intravenous drip could be chosen as the first-opted antibacterial protocol. If their susceptibility test showed resistance, they could be replaced by the sensitive one. When the infection was alleviated, treatment could be continued by a sensitive oral antibiotic. Although quinolones and aminoglycosides were sensitive to the pathogenic bacterium, they should be avoided because of their severe toxicity to infants.

In summary, simple surgical intervention like incision and drainage are recommended to YIPA. In contrast, we suggest the avoidance of active probing for the underlying fistulae and fistulotomy. ${ }^{3}$ The use of sensitive antibiotics, despite the lack of microbial-enteric flora participation, is usually justified after surgical intervention. Our experience strongly indicated that abscess incision, synchronous antibiotic administration were the optimal alternative approach which could shorten clinical course and low the incidence of abscess spread or fistula formation.

\section{Acknowledgements}

This work was supported by Research Start-up Fund for Doctors of Lishui University.

\section{REFERENCES}

1. Charalampopoulos A, Zavras N, Kapetanakis EI, et al. Surgical treatment of perianal abscess and fistulain-ano in childhood, with emphasis in children older than 2 years. J Pediatr Surg 2012; 47: 2096-2100.

2. Hanada M, Furuya T, Sugito K, et al. Evaluation of the efficacy of incision and drainage versus hainosankyuto treatment for perianal abscess in infants: A multicenter study. Surg Today 2015; 45: 1385-1389.

3. Gong Z, Han M, Wu Y, Huang X, Xu WJ, Lv Z. Treatment of first-time perianal abscess in childhood, balance recurrence and fistula formation rate with medical intervention. Eur J Pediatr Surg 2018; 28: 373-377.

4. Mamutjan A, Wen H, Huang HG, Chu H. Features and surgical management of anal abscess fistula in infants. J Med Postgra 2010; 23: 495-499.

5. Liu Y. Clinical observation of surgical treatment for infants perianal abscess and anus fistula. Chin J Clin Rational Drug Use 2012; 5: 148-149.

6. Pan YB, Gao WH, He SM. Comprehensive treatment for 115 cases of infant perianal abscess. Chin Community Doc 2012; 14: 149.

7. Inoue $\mathrm{M}$, Sugito $\mathrm{K}$, Ikeda $\mathrm{T}$, et al. Long-term results of seton placement for fistula-in-ano in infants. J Gastrointest Surg 2014; 18: 580-583.

8. Luo CL, Yu SW, Xu Z, Zhou Y, Deng KZ, Leng MM The analysis for results of the bacterial culture and drug susceptibility in patients with perianal abscess. J Colorectal Anal Surg 2013; 19: 96-98.

9. Liu X. Analyses for 58 cases of perianal abscess germiculture and susceptibility test. Chin Health Care 2010; 18: 5-7.

10. Liu CK, Liu CP, Leung $\mathrm{CH}$, Sun FJ. Clinical and microbiological analysis of adult perianal abscess. J Microbiol Immunol Infect 2011; 44: 204-208.

11. He L. Pathogens causing bacteriological perianal abscess infections and drug resistance. Chin J Nosocomiol 2012; 22: 2452-2454. 